\title{
Evaluation of the Concentration of Nutrients in the Seeds of Faba Bean (Vicia faba L. major) and PEA (Pisum sativum L.) Depending on Habitat Conditions
}

\author{
Jerzy Księżak, Jolanta Bojarszczuk*, Mariola Staniak \\ Institute of Soil Science and Plant Cultivation - State Research Institute, \\ Department of Forage Crop Production, \\ Puławy, Poland
}

Received: 12 April 2017

Accepted: 31 July 2017

\begin{abstract}
The aim of our study was to determine the effect of habitat conditions on the nutrients content in the seeds of different cultivars of faba bean and pea (edible and fodder cultivars). Experiments were located in some different parts of Poland ( 9 for faba bean, 11 for field pea). Nine cultivars of faba bean, 17 edible cultivars of pea, and 13 fodder cultivars of pea were used in these experiments. The study showed that the contents of components varied between cultivars of both species depending on examined factors. The contents of the components also varied depending on location. In southern Poland the average crude fibre and crude protein contents were higher than in the northern and northwestern parts of the country. Significant differences between cultivars of faba bean were found in the contents of crude fibre, crude protein, and soluble sugars. All the tested faba bean cultivars contained a similar amount of crude fat. The average crude protein and crude fibre content in pea seeds was higher in the fodder cultivars than in the edible ones. The smallest content of fibre among all the cultivars was noted in the northern and the largest in the southeastern regions of Poland. Most cultivars of pea showed a similar sugar content. The most favorable conditions of sugar accumulation occurred in the southern part and the least in the northeastern and the northern parts of the country. Fodder cultivars showed relatively little variability in starch content. The agro-ecological conditions influenced content of concentration of nutrients in the seeds of faba bean and pea.
\end{abstract}

Keywords: faba bean, pea, cultivar, regions, nutrients content

*e-mail: jbojarszczuk@iung.pulawy.pl 


\section{Introduction}

Production of feed protein in Europe and Poland is insufficient, which encourages us to explore the possibilities of using domestic protein raw materials to replace or supplement the imported post-extraction soy pellets. In Poland, this demand might be partially covered by legume seeds, such as faba bean and peas. However, due to differences in protein content, crude fiber, and antinutritional substances, they cannot be added to the mix in the same dosage as soybean meal [1-5]. The protein content of seed legumes ranges from about $20 \%$ in peas to $45 \%$ in yellow lupine. The level of crude fibre is conditioned by, among other factors, the thickness of the outer cove. Climatic conditions in Poland allow for the cultivation of legumes across the country [6]. They can be cultivated both in holdings with organic farming and sustainable production systems. Due to their high energy and protein content, the seeds are widely used for animal feed and food [7]. Cultivating these species in Poland is fully justified, as they improve soil fertility, improve its structure, and leave a very good position for subsequent plants. In addition, due to their ability to bind nitrogen, faba beans or peas significantly reduce the negative effects of intensive technology used in the cultivation of certain species (frequently in monoculture), thus preventing land degradation [8]. The seeds of these species can be used as the source of functional compounds [9-10]. Moreover, an increasing consumer awareness of the potential health benefits of consuming protein may stimulate the production of legumes [10-11]. The increasing demand for feed protein and expectations as regards environmental protection have spurred an expectation that the importance of these species will grow in the coming years. Their cultivation will be more and more profitable, and their seeds will become a valuable commodity.

The aim of our study was to determine the effects of habitat (agri-environmental) conditions on the content of nutrients in the seeds of faba bean and pea.

\section{Materials and Methods}

The influence of agroecological conditions on the content of nutrients was evaluated on the basis of the analysis of the material obtained from the experiments carried out in 2010-2012 at the Research Centre for Cultivar Testing (COBORU) located in different parts of Poland (Fig. 1). The experiments took into account the currently registered cultivars of faba bean: Albus, Amulet, Bobas, Granit, Kasztelan, Leo, Olga, Optimal, and Sonet; edible cultivars of pea: Akord, Batuta, Bohun, Boruta, Brylant, Cysterski, Ezop, Kavalir, Lasso, Mecenas, Medal, Mentor, Santana, Tarchalska, Terno, Wenus, Zekon; and fodder pea: Eureka, Gwarek, Hubal, Klif, Marych, Milwa, Model, Muza, Pomorska, Roch, Sokolik, Turnia, and Wiato.

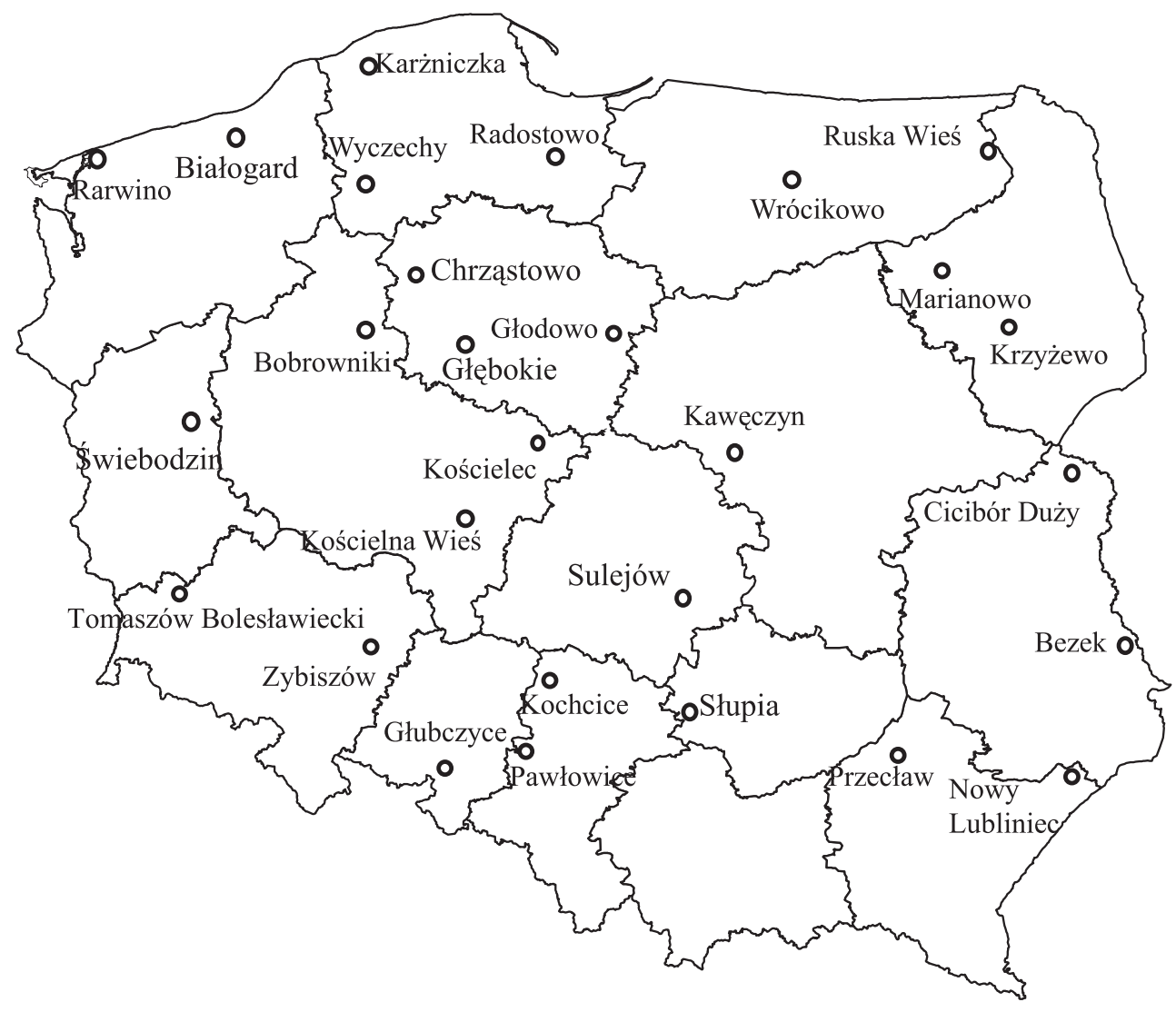

Fig. 1. Locations of experiments. 
The faba bean experiment was located in the northern and northwestern regions of Rarwino, Wrócikowo, Radostowo, and Karżniczka, and the southwestern and southeastern regions of Kochcice, Głubczyce, Pawłowice, Zybiszów, and Przecław. The locations of the pea experiment (edible cultivars) were the western and northwestern regions of Chrząstowo, Głębokie, Kościelna Wieś, and Radostowo, and the southern, central, and southeastern regions Bezek, Cicibór Duży, Kawęczyn, Krzyżewo, Pawłowice, Słupia, and Sulejów. Fodder pea locations were the northern and northwestern regions of Białogard and Wyczechy, the western regions of Bobrowniki, Świebodzin, and Tomaszów Bolesławiecki, the southern and southeastern regions of Marianowo, Pawłowice, and Ruska Wieś, the eastern region of Cicibór Duży, and the central regions of Głodowo and Kościelec.

In the first year of the study (2010) faba bean was cultivated on soils belonging to the very good wheat complex (Głubczyce, Przecław, Radostowo, Zybiszów), good wheat (Karżniczka, Pawłowice) and very good rye (Rarwino). In 2011-2012 this species was cultivated on the soil of the very good and good complex, and only in Rarwino on the soil of a very good rye complex. The soil on which faba bean was cultivated was $\mathrm{pH}$ 5.7-7.3.

In 2010 most of the edible cultivars of pea were cultivated on the soil of the good wheat complex, only in Cicibór Duży, Kawęczyn, and Krzyżewo in the soil of the very good rye complex, and in Radostowo in the soil of the very good wheat complex. Soil $\mathrm{pH}$ ranged between pH 5.5 and 7.2. In 2011 and 2012 peas were also grown on very good soils belonging to the good wheat complex (Bezek, Chrząstowo, Krzyżewo, Pawłowice, Słupia, Sulejów), very good rye (Cicibór Duży, Kawęczyn), and on the soil of the very good wheat complex (Głębokie, Radostowo).

In all years of research, fodder pea was cultivated in very good and good rye soils, and in Świebodzin in the soil of the good wheat complex. Soil $\mathrm{pH}$ ranged $\mathrm{pH}_{\mathrm{KCl}}$ 5.0-7.2.

The seeds of faba bean and pea were determined for the contents of major important nutrients: crude fibre (by weight method), crude fat (by Soxhlet's weight method), N (by flow spectrophotometry), crude ash (by weight method at $580^{\circ} \mathrm{C}$ ), and soluble sugars and starch (by Bertrand's titration method). The analyses were performed in the Main Laboratory of Chemical Analyses of IUNG-PIB in Puławy and in the laboratory of COBORU in Słupia Wielka. Total precipitation during the growing season of faba beans in individual locations (average from three years of the study) ranged 199.8-563.1 mm (Table 1), of pea (edible cultivars) 171.2-486.0 $\mathrm{mm}$ (Table 2), and of fodder pea ranged $188.6-428.9 \mathrm{~mm}$ (Table 3 ). The results were statistically analyzed with the use of the analysis of variance using Statistica v. 10.0 program (Tukey's test $\alpha=0.05$ ). The correlations between the content of nutrients in the seeds of different cultivars of faba bean and field pea and selected agrotechnical factors were also performed.

\section{Results and Discussion}

The studies have shown that regardless of the agroecological conditions, the cultivars of faba beans: Albus, Amulet, Bobas, Leo, Kasztelan, and Olga had the highest protein content, while cvs. Optimal, Sonet, and Granit had the smallest (significant differences; Table 4).

In the northern and northwestern regions of Poland, faba bean cultivars accumulated the least of protein in their seeds, while in the southern ones - the most (Table 5). According to Fordoński et al. [12], faba bean cultivars proved to the species giving the highest yields of all the tested legume crops in Northern Poland. This was confirmed by Florek et al. [13], who recorded that faba beans and peas were the most productive in the Polish conditions, while the lowest yield was obtained from yellow lupine. Sarah et al. [14] found that the content of proteins, carbohydrates, ash, fat, and fibre depends on a cultivar. In contrast, Mekkei [15] stated that regardless of a cultivar, large faba bean seeds contained more protein and carbohydrates. When assessing protein concentration in numerous cultivars, Hendawey and Younes [16] found the largest concentration of this component in the seeds of Sacha 3, Sacha 2, Sacha 4, and Missr 1, while the smallest was in Giza 3. According to these authors [16], the differences between faba bean cultivars resulted from both genetic and environmental factors. Other authors [12, 1718] have found that the nutritional value of legume seeds is variable and depends on a number of factors, among others: species and varieties of seeds and agrotechnical factors (conditions of cultivation, fertilization). Księżak [19] recorded similar concentrations of protein, fibre, fat, ash, and nitrogen-free extract compounds in faba bean cvs. Nadwiślański, Bronto, Tino, and Martin. Simultaneously, the contents of these components did not differ from the values cited in the literature [20]. According to Księżak [19], only the seeds of cv. Caspar contained fewer proteins and more nitrogen-free extract compounds. Musalam et al. [21] studied the chemical composition of faba bean seeds under soil irrigation conditions. They found that such seeds had higher protein, ash, and fibre contents, but significantly lower fat and carbohydrate contents. Sarah et al. [14] showed significant differences in protein content among the seeds of faba bean cultivars.

Simultaneously, these authors recorded almost twofold higher digestibility of cooked protein compared to raw. Fordoński et al. [12] found that protein content in faba bean seed ranged from 28.6 to $29.5 \%$ of dry matter. Much the same protein content in faba bean seeds were obtained in long-term research carried out by the Research Centre for Cultivar Testing [22]. They also found less protein content in faba bean seeds compared to those attached in NŻ́́ [23]. Faba bean seeds of all varieties had lower crude protein content compared to the content determined in the tested cultivar (Albus) by Szpunar-Krok et al. [24], but the figure was close to that reported by Hanczakowska and Świątkiewicz [25].

In our own studies, the cultivars exhibited a diverse fibre content, with its lowest amount being recorded for 
Table 1. Monthly sum of precipitation and mean day and night temperatures in locations of cultivation of faba bean (2010-2012).

\begin{tabular}{|c|c|c|c|c|c|c|c|c|c|c|}
\hline \multirow[b]{2}{*}{ Location } & \multicolumn{5}{|c|}{ Precipitation (mm) } & \multicolumn{5}{|c|}{ Temperature $\left({ }^{\circ} \mathrm{C}\right)$} \\
\hline & IV & $\mathrm{V}$ & VI & VII & $\begin{array}{c}\text { Sum } \\
\text { (IV-VII) }\end{array}$ & IV & $\mathrm{V}$ & VI & VII & $\begin{array}{l}\text { Average } \\
\text { (IV-VII) }\end{array}$ \\
\hline \multicolumn{11}{|c|}{2010} \\
\hline Głubczyce & 58.2 & 193.2 & 103.2 & 208.5 & 563.1 & 7.6 & 11.8 & 16.7 & 20.4 & 14.1 \\
\hline Karżniczka & 9.8 & 112.4 & 16.4 & 142.4 & 281.0 & 6.8 & 9.7 & 15.0 & 19.7 & 12.8 \\
\hline Kochcice & - & - & - & - & - & - & - & - & - & - \\
\hline Pawłowice & 54.3 & 214.6 & 61.2 & 21.0 & 351.1 & 8.7 & 12.4 & 17.1 & 97.6 & 34.0 \\
\hline Przecław & 50.5 & 170.0 & 100.9 & 174.2 & 495.6 & 8.9 & 14.2 & 18.3 & 20.8 & 15.6 \\
\hline Radostowo & 7.8 & 92.4 & 41.8 & 77.3 & 219.3 & 7.2 & 11.2 & 15.3 & 20.5 & 13.6 \\
\hline Rarwino & 20.0 & 85.1 & 27.7 & 74.8 & 207.6 & 6.5 & 8.7 & 14.1 & 20.0 & 12.3 \\
\hline Wrócikowo & 16.9 & 153.0 & 80.8 & 64.1 & 314.8 & 7.3 & 11.9 & 15.8 & 20.5 & 13.9 \\
\hline Zybiszów & 34.5 & 118.1 & 32.4 & 109.7 & 294.7 & 9.2 & 12.7 & 17.9 & 21.1 & 15.2 \\
\hline \multicolumn{11}{|c|}{2011} \\
\hline Głubczyce & 28.8 & 43.4 & 99.5 & 167.5 & 339.2 & 9.7 & 14.2 & 17.4 & 17.4 & 14.7 \\
\hline Karżniczka & 24.0 & 42.0 & 75.2 & 100.4 & 241.6 & 9.3 & 12.5 & 16.5 & 17.7 & 14.0 \\
\hline Kochcice & 16.0 & 65.4 & 90.8 & 132.5 & 304.7 & 10.3 & 13.5 & 17.8 & 16.6 & 14.6 \\
\hline Pawłowice & 21.4 & 57.4 & 42.8 & 111.2 & 232.8 & 10.4 & 13.6 & 18.3 & 17.6 & 15.0 \\
\hline Przecław & 51.8 & 38.1 & 71.3 & 291.8 & 453.0 & 10.0 & 23.5 & 18.1 & 18.5 & 17.5 \\
\hline Radostowo & 17.2 & 32.1 & 36.2 & 114.3 & 199.8 & 9.1 & 12.5 & 17.2 & 18.2 & 14.3 \\
\hline Rarwino & 12.1 & 52.7 & 62.9 & 175.2 & 302.9 & 10.1 & 12.7 & 17.0 & 16.9 & 14.2 \\
\hline Wrócikowo & 26.6 & 40.9 & 63.2 & 203.4 & 334.1 & 9.2 & 12.7 & 17.0 & 17.6 & 14.1 \\
\hline Zybiszów & 32.7 & 56.3 & 48.2 & 206.3 & 343.5 & 11.5 & 13.7 & 18.7 & 18.1 & 15.5 \\
\hline \multicolumn{11}{|c|}{2012} \\
\hline Głubczyce & 54.0 & 29.3 & 97.8 & 66.6 & 247.7 & 8.3 & 14.8 & 17.4 & 19.9 & 15.1 \\
\hline Karżniczka & 42.1 & 18.9 & 123.7 & 172.2 & 356.9 & 7.6 & 4.6 & 14.7 & 18.0 & 11.2 \\
\hline Kochcice & 43.2 & 41.4 & 73.8 & 68.4 & 226.8 & 9.0 & 14.5 & 17.2 & 19.6 & 15.1 \\
\hline Pawłowice & 64.8 & 35.2 & 70.7 & 50.9 & 221.6 & 9.0 & 15.9 & 17.8 & 20.9 & 15.9 \\
\hline Przecław & 21.7 & 66.7 & 66.9 & 65.6 & 220.9 & 9.9 & 14.8 & 17.7 & 20.9 & 15.8 \\
\hline Radostowo & 34.5 & 28.6 & 136.1 & 89.9 & 289.1 & 8.0 & 13.6 & 15.1 & 18.1 & 13.7 \\
\hline Rarwino & 39.8 & 12.8 & 83.0 & 161.1 & 296.7 & 7.1 & 12.5 & 15.1 & 17.3 & 13.0 \\
\hline Wrócikowo & 79.5 & 48.5 & 97.6 & 106.0 & 331.6 & 7.8 & 13.3 & 15.1 & 18.9 & 13.8 \\
\hline Zybiszów & 33.1 & 34.7 & 81.7 & 101.6 & 251.1 & 9.6 & 15.4 & 17.3 & 20.2 & 15.6 \\
\hline
\end{tabular}

Source: own elaboration based on IMGW-PIB data [2010, 2011, 2012]

Bobas and Granit. The region of cultivation also affected the contents of that component in faba bean. It was the least accumulated by the seeds collected in the northern part, and the most in the southern parts of Poland (Table $5)$. The contents of crude fibre were similar to the levels reported by Kiarie et al. [26].

The amount of crude fat was similar in all the evaluated cultivars (insignificant differences). When assessing fat content in seeds of faba bean cultivars, Hendawey and Younes [16] found its higher concentration in cvs. Giza 843 and Giza 3. In our study a higher fat concentration in faba bean seeds was recorded in the northern part of Poland and lower in the south. Pisulewska et al. [20] found a strong relationship between the fat content in the seeds and weather conditions during the growing season. These authors undermine the thesis 
Table 2. Monthly sum of precipitation and mean day and night temperature in locations of cultivation of field edible pea cultivars (20102012).

\begin{tabular}{|c|c|c|c|c|c|c|c|c|c|c|}
\hline \multirow[b]{2}{*}{ Location } & \multicolumn{5}{|c|}{ Precipitation (mm) } & \multicolumn{5}{|c|}{ Temperature $\left({ }^{\circ} \mathrm{C}\right)$} \\
\hline & IV & $\mathrm{V}$ & VI & VII & $\begin{array}{c}\text { Sum } \\
\text { (IV-VII) }\end{array}$ & IV & $\mathrm{V}$ & VI & VII & $\begin{array}{l}\text { Average } \\
\text { (IV-VII) }\end{array}$ \\
\hline \multicolumn{11}{|c|}{2010} \\
\hline Bezek & 18.9 & 72.4 & 94.4 & 156.3 & 342.0 & 9.0 & 14.5 & 17.6 & 21.6 & 15.7 \\
\hline Cicibór Duży & 23.0 & 126.8 & 67.7 & 48.2 & 265.7 & 8.7 & 14.5 & 17.9 & 21.6 & 15.7 \\
\hline Chrząstowo & 35.4 & 119.7 & 9.5 & 147.9 & 312.5 & 7.8 & 11.3 & 16.6 & 21.7 & 14.4 \\
\hline Głębokie & 32.4 & 35.4 & 27.2 & 162.4 & 257.4 & 8.3 & 12.5 & 16.8 & 21.5 & 14.8 \\
\hline Kawęczyn & 15.1 & 155.5 & 37.0 & 67.4 & 275.0 & 8.9 & 13.4 & 17.1 & 21.5 & 15.2 \\
\hline Krzyżewo & 29.8 & 148.0 & 74.1 & 87.0 & 338.9 & 8.0 & 14.0 & 17.2 & 21.3 & 15.1 \\
\hline Pawłowice & 54.3 & 214.6 & 61.2 & 97.6 & 427.7 & 8.7 & 12.3 & 17.1 & 21.1 & 14.8 \\
\hline Radostowo & 7.9 & 92.4 & 41.8 & 77.3 & 219.4 & 7.2 & 11.5 & 15.3 & 19.7 & 13.4 \\
\hline Słupia & 40.8 & 197.8 & 78.5 & 168.9 & 486.0 & 8.1 & 12.8 & 16.5 & 20.1 & 14.4 \\
\hline Sulejów & 25.5 & 136.9 & 58.3 & 86.7 & 307.4 & 8.3 & 12.8 & 16.9 & 20.7 & 14.7 \\
\hline \multicolumn{11}{|c|}{2011} \\
\hline Bezek & 30.6 & 40.8 & 80.0 & 178.9 & 330.3 & 9.9 & 14.6 & 18.3 & 19.4 & 15.6 \\
\hline Cicibór Duży & 37.6 & 61.0 & 65.4 & 201.6 & 365.6 & 9.5 & 13.7 & 18.4 & 18.1 & 14.9 \\
\hline Chrząstowo & 9.0 & 38.4 & 39.7 & 115.5 & 202.6 & 10.9 & 13.7 & 18.6 & 17.8 & 15.3 \\
\hline Głębokie & 6.4 & 39.7 & 87.5 & 95.2 & 228.8 & 11.2 & 14.7 & 18.1 & 18.0 & 15.5 \\
\hline Kawęczyn & 40.2 & 31.5 & 51.4 & 179.8 & 302.9 & 10.6 & 14.3 & 18.6 & 18.8 & 15.6 \\
\hline Krzyżewo & 42.5 & 66.4 & 44.6 & 218.0 & 371.5 & 9.8 & 13.3 & 18.0 & 19.2 & 15.1 \\
\hline Pawłowice & 21.4 & 57.4 & 42.8 & 111.0 & 232.6 & 10.4 & 13.4 & 19.3 & 17.7 & 15.2 \\
\hline Radostowo & 17.2 & 32.1 & 36.2 & 114.3 & 199.8 & 9.3 & 12.4 & 16.8 & 18.2 & 14.2 \\
\hline Słupia & 29.4 & 49.7 & 25.6 & 166.9 & 271.6 & 9.7 & 13.9 & 18.4 & 17.5 & 14.9 \\
\hline Sulejów & 21.6 & 49.8 & 22.6 & 175.5 & 269.5 & 9.8 & 13.5 & 18.1 & 18.2 & 14.9 \\
\hline \multicolumn{11}{|c|}{2012} \\
\hline Bezek & 33.4 & 40.3 & 66.5 & 31.0 & 171.2 & 9.3 & 15.1 & 17.2 & 21.5 & 15.8 \\
\hline Cicibór Duży & 37.5 & 55.8 & 126.1 & 27.5 & 246.9 & 8.9 & 14.8 & 16.9 & 21.0 & 15.4 \\
\hline Chrząstowo & 30.4 & 42.9 & 113.9 & 144.3 & 331.5 & 8.8 & 14.6 & 15.6 & 18.9 & 14.5 \\
\hline Głębokie & 16.3 & 29.3 & 103.7 & 68.8 & 218.1 & 9.3 & 15.3 & 16.1 & 19.6 & 15.1 \\
\hline Kawęczyn & 46.0 & 46.5 & 67.3 & 74.3 & 234.1 & 9.6 & 15.4 & 17.0 & 20.6 & 15.7 \\
\hline Krzyżewo & 40.4 & 46.5 & 87.3 & 91.7 & 265.9 & 8.2 & 14.1 & 15.5 & 19.8 & 14.4 \\
\hline Pawłowice & 64.8 & 35.2 & 70.7 & 50.9 & 221.6 & 9.0 & 15.9 & 17.8 & 20.9 & 15.9 \\
\hline Radostowo & 34.5 & 28.6 & 136.1 & 89.9 & 289.1 & 8.0 & 13.6 & 15.1 & 18.1 & 13.7 \\
\hline Słupia & 55.7 & 17.3 & 100.0 & 81.3 & 254.3 & 8.9 & 14.5 & 17.4 & 20.2 & 15.3 \\
\hline Sulejów & 41.5 & 22.7 & 69.0 & 60.3 & 193.5 & 8.8 & 14.7 & 17.0 & 20.5 & 15.3 \\
\hline
\end{tabular}

Source: own elaboration based on IMGW-PIB data [2010, 2011, 2012]

provided in the literature that the chemical composition of faba bean seeds depends little on environmental factors. In our own study we found that the highest soluble sugar content was recorded for cv. Leo, while the lowest was for cvs. Albus and Bobas (significant differences). The differences in chemical composition 
Table 3. Monthly sum of precipitation and mean day and night temperatures in locations of fodder pea cultivation (2010-2012).

\begin{tabular}{|c|c|c|c|c|c|c|c|c|c|c|}
\hline \multirow{2}{*}{ Location } & \multicolumn{5}{|c|}{ Precipitation (mm) } & \multicolumn{5}{|c|}{ Temperature $\left({ }^{\circ} \mathrm{C}\right)$} \\
\hline & IV & $\mathrm{V}$ & VI & VII & $\begin{array}{c}\text { Sum } \\
\text { (IV-VII) }\end{array}$ & IV & $\mathrm{V}$ & VI & VII & $\begin{array}{l}\text { Average } \\
\text { (IV-VII) }\end{array}$ \\
\hline \multicolumn{11}{|c|}{2010} \\
\hline Białogard & 11.7 & 95.6 & 58.3 & 114.7 & 280.3 & 7.3 & 10.1 & 15.6 & 20.6 & 13.4 \\
\hline Bobrowniki & 37.3 & 83.1 & 12.5 & 95.6 & 228.5 & 7.9 & 11.2 & 16.5 & 21.5 & 14.3 \\
\hline Cicibór Duży & 23.0 & 126.8 & 67.7 & 48.2 & 265.7 & 8.7 & 14.5 & 17.9 & 21.6 & 15.7 \\
\hline Głodowo & 29.2 & 143.3 & 44.7 & 130.7 & 347.9 & 8.5 & 12.5 & 16.8 & 21.4 & 14.8 \\
\hline Kościelec & 25.0 & 147.7 & 41.3 & 87.5 & 301.5 & 10.2 & 13.5 & 19.0 & 23.1 & 16.5 \\
\hline Marianowo & 34.8 & 151.8 & 75.5 & 108.1 & 370.2 & 8.3 & 13.4 & 16.9 & 21.1 & 14.9 \\
\hline Pawłowice & 54.3 & 214.6 & 61.2 & 21.0 & 351.1 & 8.7 & 12.4 & 17.1 & 97.6 & 34.0 \\
\hline Ruska Wieś & 42.8 & 140.1 & 125.9 & 94.9 & 403.7 & 7.0 & 12.8 & 16.1 & 20.7 & 14.2 \\
\hline Świebodzin & 22.8 & 84.0 & 13.1 & 68.7 & 188.6 & 8.7 & 11.4 & 17.3 & 21.7 & 14.8 \\
\hline $\begin{array}{c}\text { Tomaszów } \\
\text { Bolesławiecki }\end{array}$ & 36.9 & 105.5 & 35.6 & 73.7 & 251.7 & 8.1 & 11.7 & 16.2 & 20.8 & 14.2 \\
\hline Wyczechy & 54.3 & 214.6 & 61.2 & 97.6 & 427.7 & 8.7 & 12.4 & 17.1 & 21.0 & 14.8 \\
\hline \multicolumn{11}{|c|}{2011} \\
\hline Białogard & 13.8 & 38.2 & 63.6 & 109.8 & 225.4 & 10.0 & 13.2 & 17.1 & 17.4 & 14.4 \\
\hline Bobrowniki & 5.0 & 32.7 & 65.2 & 152.2 & 255.1 & 11.4 & 14.9 & 18.3 & 17.8 & 15.6 \\
\hline Cicibór Duży & 37.6 & 61.2 & 65.4 & 201.6 & 365.8 & 9.6 & 13.8 & 18.4 & 18.7 & 15.1 \\
\hline Głodowo & 12.4 & 43.4 & 36.2 & 179.3 & 271.3 & 10.4 & 14.2 & 18.2 & 18.1 & 15.2 \\
\hline Kościelec & 16.5 & 50.2 & 51.6 & 117.0 & 235.3 & 12.3 & 16.4 & 21.0 & 19.9 & 17.4 \\
\hline Marianowo & 38.7 & 58.0 & 58.3 & 273.9 & 428.9 & 9.6 & 13.4 & 17.7 & 18.5 & 14.8 \\
\hline Pawłowice & 21.4 & 57.4 & 42.8 & 111.2 & 232.8 & 10.4 & 13.6 & 18.3 & 17.6 & 15.0 \\
\hline Ruska Wieś & 35.7 & 67.1 & 43.9 & 191.6 & 338.3 & 8.7 & 12.6 & 17.1 & 18.4 & 14.2 \\
\hline Świebodzin & 14.9 & 20.5 & 37.2 & 143,0 & 215.6 & 11.6 & 14.5 & 18.6 & 17.8 & 15.6 \\
\hline $\begin{array}{c}\text { Tomaszów } \\
\text { Bolesławiecki }\end{array}$ & 25.0 & 48.0 & 63.5 & 187.3 & 323.8 & 10.7 & 13.6 & 18.2 & 17.5 & 15.0 \\
\hline Wyczechy & 14.1 & 50.6 & 55.9 & 166.8 & 287.4 & 10.2 & 13.1 & 17.2 & 17.4 & 14.5 \\
\hline \multicolumn{11}{|c|}{2012} \\
\hline Białogard & 31.6 & 24.7 & 104.2 & 102.2 & 262.7 & 8.0 & 13.7 & 15.6 & 18.2 & 13.9 \\
\hline Bobrowniki & 30.0 & 40.4 & 90.8 & 127.5 & 288.7 & 8.6 & 14.8 & 16.5 & 19.0 & 14.7 \\
\hline Cicibór Duży & 37.5 & 55.8 & 126.1 & 27.5 & 246.9 & 8,9 & 14.8 & 16.9 & 21.0 & 15.4 \\
\hline Głodowo & 34.9 & 17.9 & 124.4 & 89.7 & 266.9 & 9.1 & 15.4 & 16.1 & 20.9 & 15.4 \\
\hline Kościelec & 11.1 & 32.8 & 121.5 & 100.5 & 265.9 & 9.5 & 13.8 & 18.5 & 21.9 & 15.9 \\
\hline Marianowo & 44.6 & 60.3 & 105.5 & 101.1 & 311.5 & 8.5 & 14.1 & 15.5 & 19.7 & 14.5 \\
\hline Pawłowice & 64.8 & 35.2 & 70.7 & 50.9 & 221.6 & 9.0 & 15.9 & 17.8 & 20.9 & 15.9 \\
\hline Ruska Wieś & 64.5 & 65.6 & 99.1 & 126.8 & 356.0 & 7.1 & 13.0 & 13.4 & 18.9 & 13.1 \\
\hline Świebodzin & 32.6 & 54.8 & 79.3 & 154.4 & 321.1 & 9.4 & 15.5 & 16.5 & 19.3 & 15.2 \\
\hline $\begin{array}{c}\text { Tomaszów } \\
\text { Bolesławiecki }\end{array}$ & 45.5 & 45.8 & 74.8 & 118.1 & 284.2 & 8.5 & 14.4 & 16.1 & 18.8 & 14.5 \\
\hline Wyczechy & 53.8 & 24.3 & 128.3 & 111.7 & 318.1 & 7.9 & 13.8 & 15.0 & 18.4 & 13.8 \\
\hline
\end{tabular}

Source: own elaboration based on IMGW-PIB data [2010, 2011, 2012] 
Table 4. Nutrient contents in faba bean depending on the cultivar $(\% \mathrm{DM})$.

\begin{tabular}{|c|c|c|c|c|}
\hline Cultivar & $\begin{array}{c}\text { Crude } \\
\text { protein }\end{array}$ & $\begin{array}{c}\text { Crude } \\
\text { fibre }\end{array}$ & $\begin{array}{c}\text { Crude } \\
\text { fat }\end{array}$ & Sugars \\
\hline Albus & $28.28^{\text {b }}$ & $7.15^{\mathrm{b}}$ & $1.44^{\mathrm{a}}$ & $4.58^{\mathrm{a}}$ \\
\hline Amulet & $28.41^{\mathrm{b}}$ & $7.11^{\mathrm{b}}$ & $1.47^{\mathrm{a}}$ & $4.97^{\mathrm{bc}}$ \\
\hline Bobas & $28.47^{\mathrm{b}}$ & $6.42^{\mathrm{a}}$ & $1.45^{\mathrm{a}}$ & $4.56^{\mathrm{a}}$ \\
\hline Granit & $26.46^{\mathrm{a}}$ & $6.49^{\mathrm{a}}$ & $1.54^{\mathrm{a}}$ & $4.69^{\mathrm{ab}}$ \\
\hline Kasztelan & $27.86^{\mathrm{b}}$ & $7.20^{\mathrm{b}}$ & $1.43^{\mathrm{a}}$ & $4.80^{\mathrm{abc}}$ \\
\hline Leo & $28.29^{\mathrm{b}}$ & $6.86^{\mathrm{ab}}$ & $1.52^{\mathrm{a}}$ & $5.06^{\mathrm{c}}$ \\
\hline Olga & $27.92^{\mathrm{b}}$ & $7.01^{\mathrm{b}}$ & $1.57^{\mathrm{a}}$ & $4.90^{\mathrm{abc}}$ \\
\hline Optimal & $26.51^{\mathrm{a}}$ & $6.97^{\mathrm{b}}$ & $1.52^{\mathrm{a}}$ & $4.78^{\mathrm{abc}}$ \\
\hline Sonet & $26.40^{\mathrm{a}}$ & $6.77^{\mathrm{ab}}$ & $1.46^{\mathrm{a}}$ & $4.59^{\mathrm{abc}}$ \\
\hline Mean & $\mathbf{2 7 . 6 4}$ & $\mathbf{6 . 8 9}$ & $\mathbf{1 . 4 9}$ & 4.77 \\
\hline
\end{tabular}

*values in column marked the same letter did not differ statistically $(\alpha=0.05)$

between faba bean cultivars were also noted by Abusin et al. [27] and El-Saber [28].

Correlation analysis showed that the content of proteins in faba bean seeds was negatively affected by the contents of fibre and tannins (significant differences), but it was positively affected by crude fat (Table 6). In previous studies, Księżak [19] found a negative correlation between protein content in the seeds and the contents of ash, nitrogen-free compounds, potassium, and magnesium, but a positive correlation between the content of nitrogen-free extract compounds and 1,000 seed weight. These relationships were confirmed in the study of Jasińska and Kotecki [29]. An increase of crude fibre content in faba bean seeds was significantly affected

Table 5. Nutrient contents in faba bean depending on the cultivation region $(\% \mathrm{DM})$.

\begin{tabular}{|c|c|c|c|c|}
\hline Location & $\begin{array}{c}\text { Crude } \\
\text { protein }\end{array}$ & $\begin{array}{c}\text { Crude } \\
\text { fibre }\end{array}$ & $\begin{array}{c}\text { Crude } \\
\text { fat }\end{array}$ & Sugars \\
\hline Głubczyce & $28.14^{\text {*def }}$ & $6.82^{\text {cd }}$ & $1.53^{\text {abcd }}$ & $4.76^{\mathrm{a}}$ \\
\hline Karżniczka & $27.49^{\text {bcd }}$ & $6.32^{\mathrm{a}}$ & $1.78^{\mathrm{d}}$ & $4.86^{\mathrm{a}}$ \\
\hline Kochcice & $27.74^{\text {cde }}$ & $7.74^{\mathrm{e}}$ & $1.31^{\mathrm{a}}$ & $4.82^{\mathrm{a}}$ \\
\hline Pawłowice & $28.51^{\text {ef }}$ & $6.98^{\mathrm{d}}$ & $1.42^{\mathrm{ab}}$ & $4.79^{\mathrm{a}}$ \\
\hline Przecław & $29.20^{\mathrm{f}}$ & $7.07^{\mathrm{cd}}$ & $1.30^{\mathrm{ab}}$ & $4.94^{\mathrm{a}}$ \\
\hline Radostowo & $26.97^{\mathrm{abc}}$ & $6.57^{\mathrm{bc}}$ & $1.59^{\mathrm{bcd}}$ & $4.67^{\mathrm{a}}$ \\
\hline Rarwino & $26.39^{\mathrm{a}}$ & $6.60^{\mathrm{bc}}$ & $1.66^{\mathrm{cd}}$ & $4.69^{\mathrm{a}}$ \\
\hline Wrócikowo & $26.42^{\mathrm{ab}}$ & $6.95^{\mathrm{bc}}$ & $1.54^{\text {abcd }}$ & $4.75^{\mathrm{a}}$ \\
\hline Zybiszów & $28.77^{\text {def }}$ & $6.96^{\mathrm{cd}}$ & $1.56^{\text {bcd }}$ & $4.80^{\mathrm{a}}$ \\
\hline Mean & $\mathbf{2 7 . 7 1}$ & $\mathbf{6 . 8 9}$ & $\mathbf{1 . 5 2}$ & $\mathbf{4 . 7 9}$ \\
\hline
\end{tabular}

*see the explanation under Table 1
Table 6. Correlation coefficients between nutrient contents in faba bean seeds and some agrotechnical factors.

\begin{tabular}{|c|c|c|c|c|c|}
\hline & $\begin{array}{c}\text { Crude } \\
\text { protein }\end{array}$ & $\begin{array}{c}\text { Crude } \\
\text { fibre }\end{array}$ & $\begin{array}{c}\text { Crude } \\
\text { fat }\end{array}$ & Tannins & Sugars \\
\hline Crude fibre & $\mathbf{- 0 . 1 7 7}$ & & & & \\
\hline Crude fat & 0.019 & -0.193 & & & \\
\hline Tannins & $\mathbf{- 0 . 3 8 7}$ & -0.208 & -0.040 & & \\
\hline Sugars & -0.056 & 0.214 & $\mathbf{0 . 1 7 5}$ & $\mathbf{- 0 . 1 9 3}$ & \\
\hline Soil complex & $\mathbf{- 0 . 6 6 2}$ & $\mathbf{- 0 , 0 5 4}$ & $\mathbf{0 . 4 2 1}$ & -0.02 & -0.185 \\
\hline Soil pH & $\mathbf{- 0 . 3 2 2}$ & $\mathbf{0 . 0 9 9}$ & -0.024 & 0.022 & 0.031 \\
\hline Forecrop & $\mathbf{- 0 . 4 6 2}$ & $\mathbf{0 . 2 1 4}$ & 0,171 & 0.360 & -0.069 \\
\hline Precipitations & 0.106 & 0.253 & $\mathbf{- 0 . 1 5 6}$ & 0.025 & 0.120 \\
\hline
\end{tabular}

*- bold letters means significant differences

by the amount of precipitation during the growing season and the content of soluble sugars. On the other hand, its amount was reduced by the concentration of tannins and crude fat. Crude fat concentration was negatively correlated with the amount of precipitation while being positively correlated with soluble sugars (significant

Table 7. Nutrient contents in edible pea depending on the cultivar (\% DM).

\begin{tabular}{|c|c|c|c|c|}
\hline Cultivar & $\begin{array}{c}\text { Crude } \\
\text { protein }\end{array}$ & $\begin{array}{c}\text { Crude } \\
\text { fibre }\end{array}$ & Sugars & Starch \\
\hline Akord & $21.47^{\text {abc }}$ & $5.46^{\text {bcd }}$ & $6.29^{\text {abc }}$ & $44.62^{\text {bcdef }}$ \\
\hline Batuta & $22.15^{\text {abc }}$ & $5.38^{\text {bc }}$ & $6.67^{\text {bc }}$ & $44.79^{\text {bcdef }}$ \\
\hline Bohun & $21.62^{\mathrm{ab}}$ & $5.79^{\mathrm{d}}$ & $6,43^{\mathrm{abc}}$ & $47.18^{\text {ef }}$ \\
\hline Boruta & $21.70^{\mathrm{ab}}$ & $5.28^{\mathrm{abc}}$ & $6.37^{\mathrm{abc}}$ & $41.96^{\mathrm{abcd}}$ \\
\hline Brylant & $21.78^{\mathrm{abc}}$ & $5.20^{\mathrm{abc}}$ & $6.35^{\mathrm{abc}}$ & $46.67^{\text {cdef }}$ \\
\hline Cysterski & $21.58^{\mathrm{a}}$ & $5.49^{\mathrm{d}}$ & $6,19^{\mathrm{abc}}$ & $44.22^{\mathrm{bcde}}$ \\
\hline Ezop & $22.72^{\mathrm{c}}$ & $5.34^{\mathrm{bc}}$ & $6.27^{\mathrm{abc}}$ & $48.75^{\mathrm{f}}$ \\
\hline Kavalir & $22.57^{\mathrm{ab}}$ & $5.14^{\mathrm{ab}}$ & $5.96^{\mathrm{ab}}$ & $40.95^{\mathrm{abc}}$ \\
\hline Lasso & $21.45^{\mathrm{a}}$ & $5.22^{\mathrm{abc}}$ & $6.53^{\mathrm{bc}}$ & $49.19^{\mathrm{f}}$ \\
\hline Mecenas & $21.96^{\mathrm{abc}}$ & $4.87^{\mathrm{a}}$ & $6.72^{\mathrm{bcd}}$ & $46.85^{\text {defg }}$ \\
\hline Medal & $21.70^{\mathrm{ab}}$ & $5.28^{\mathrm{abc}}$ & $6.21^{\mathrm{abc}}$ & $46.15^{\text {def }}$ \\
\hline Mentor & $21.54^{\mathrm{ab}}$ & $5.32^{\mathrm{abc}}$ & $6.32^{\mathrm{abc}}$ & $47.81^{\mathrm{fg}}$ \\
\hline Santana & $22.18^{\mathrm{abc}}$ & $5.52^{\mathrm{cd}}$ & $6.53^{\mathrm{bc}}$ & $41.20^{\mathrm{abc}}$ \\
\hline Tarchalska & $21.50^{\mathrm{a}}$ & $5.36^{\mathrm{bc}}$ & $6.03^{\mathrm{ab}}$ & $47.60^{\mathrm{fg}}$ \\
\hline Terno & $22.05^{\mathrm{abc}}$ & $5.25^{\mathrm{abc}}$ & $6.54^{\mathrm{bc}}$ & $37.12^{\mathrm{a}}$ \\
\hline Wenus & $21.65^{\mathrm{ab}}$ & $5.23^{\mathrm{abc}}$ & $5.69^{\mathrm{a}}$ & $47.03^{\mathrm{fg}}$ \\
\hline Zekon & $22.30^{\mathrm{abc}}$ & $5.48^{\mathrm{bc}}$ & $6.74^{\mathrm{d}}$ & $39.47^{\mathrm{ab}}$ \\
\hline $\mathbf{2 1 . 8 8}$ & $\mathbf{5 . 3 3}$ & $\mathbf{6 . 3 4}$ & $\mathbf{4 4 . 8 1}$ \\
\hline
\end{tabular}

*see the explanation under Table 1 
Table 8. Nutrient contents in edible cultivars of field pea depending on the cultivation region (\% DM).

\begin{tabular}{|c|c|c|c|c|}
\hline Location & $\begin{array}{c}\text { Crude } \\
\text { protein }\end{array}$ & $\begin{array}{c}\text { Crude } \\
\text { fibre }\end{array}$ & Sugars & Starch \\
\hline Bezek & $22.84^{\text {*d }}$ & $5.82^{\mathrm{b}}$ & $6.70^{\mathrm{c}}$ & $47.25^{\mathrm{d}}$ \\
\hline Chrząstowo & $20.94^{\mathrm{a}}$ & $4.70^{\mathrm{a}}$ & $6.14^{\mathrm{ab}}$ & - \\
\hline Cicibór Duży & $22.75^{\mathrm{cd}}$ & $6.19^{\mathrm{c}}$ & $6.64^{\mathrm{bc}}$ & $45.51^{\mathrm{bc}}$ \\
\hline Głębokie & $21.94^{\mathrm{b}}$ & $5.07^{\mathrm{ab}}$ & $6.38^{\mathrm{abc}}$ & - \\
\hline Kawęczyn & $22.76^{\mathrm{cd}}$ & $5.73^{\mathrm{b}}$ & $6.48^{\mathrm{bc}}$ & - \\
\hline $\begin{array}{c}\text { Kościelna } \\
\text { Wieś }\end{array}$ & $18.15^{\mathrm{a}}$ & $4.80^{\mathrm{a}}$ & $6.09^{\mathrm{ab}}$ & - \\
\hline Krzyżewo & $22.68^{\mathrm{bc}}$ & $4.78^{\mathrm{a}}$ & $5.88^{\mathrm{a}}$ & - \\
\hline Pawłowice & $23.48^{\mathrm{d}}$ & $6.12^{\mathrm{c}}$ & $6.50^{\mathrm{bc}}$ & $45.11^{\mathrm{b}}$ \\
\hline Radostowo & $22.49^{\mathrm{bc}}$ & $4.79^{\mathrm{a}}$ & $5.90^{\mathrm{a}}$ & $41.31^{\mathrm{a}}$ \\
\hline Słupia & $22.29^{\mathrm{bc}}$ & $4.86^{\mathrm{a}}$ & $6.24^{\mathrm{abc}}$ & - \\
\hline Sulejów & $23.01^{\mathrm{cd}}$ & $5.76^{\mathrm{b}}$ & $6.42^{\mathrm{abc}}$ & - \\
\hline Mean & $\mathbf{2 2 . 1 2}$ & $\mathbf{5 . 3 3}$ & $\mathbf{6 . 3 1}$ & $\mathbf{4 4 . 8 0}$ \\
\hline
\end{tabular}

*see the explanation under Table 1

correlations). According to Rybiński et al. [30], the increase of fat content increases the percentage of less desirable saturated acids and of favorable oleic acid, while significantly reducing the amount of linolenic acid. Therefore, according to this author a balanced fatty acid profile is even more important than the increase in fat content. The amount of tannins was negatively correlated with the content of soluble sugars. Soil complex positively affected the content of crude fat and negatively affected crude protein. Soil $\mathrm{pH}$ and forecrop has a negative impact on crude protein content in faba bean seeds. Increasing the sum of precipitation caused an increase of crude fibre content and a decrease in crude fat content.

The study showed that regardless of agro-ecological conditions or the cultivation location of pea, the variability

Table 9. Correlation coefficients between nutrient contents in edible cultivars of pea seeds and some agrotechnical factors.

\begin{tabular}{|c|c|c|c|c|}
\hline & $\begin{array}{c}\text { Crude } \\
\text { protein }\end{array}$ & $\begin{array}{c}\text { Crude } \\
\text { fibre }\end{array}$ & Sugars & Starch \\
\hline Crude fibre & 0.0400 & & & \\
\hline Sugars & -0.1393 & $\mathbf{- 0 . 1 0 8 5}$ & & \\
\hline Starch & 0.1761 & 0.0697 & $\mathbf{- 0 . 5 6 3 4}$ & \\
\hline Soil complex & $\mathbf{0 . 1 6 4 6}$ & -0.0642 & -0.0738 & \\
\hline Soil pH & -0.0145 & $\mathbf{- 0 . 1 8 6 5}$ & $\mathbf{0 . 2 2 1 7}$ & 0.0358 \\
\hline Forecrop & $\mathbf{0 . 1 0 9 1}$ & -0.0015 & $\mathbf{0 . 1 3 9 7}$ & -0.0193 \\
\hline Precipitations & 0.0499 & $\mathbf{- 0 . 1 3 6 8}$ & $\mathbf{- 0 . 1 8 7 8}$ & $\mathbf{- 0 . 2 7 0 5}$ \\
\hline
\end{tabular}

Bold letters means significant differences
Table 10. Nutrient contents in fodder pea depending on the cultivar $(\% \mathrm{DM})$.

\begin{tabular}{|c|c|c|c|c|}
\hline Cultivar & $\begin{array}{c}\text { Crude } \\
\text { protein }\end{array}$ & $\begin{array}{c}\text { Crude } \\
\text { fibre }\end{array}$ & Sugars & Starch \\
\hline Eureka & $23.62^{\text {bcd }}$ & $5.48^{\mathrm{ab}}$ & $6.04^{\mathrm{abc}}$ & $46.65^{\mathrm{b}}$ \\
\hline Gwarek & $24.42^{\text {cdef }}$ & $5.33^{\mathrm{a}}$ & $6.24^{\mathrm{c}}$ & $46.40^{\mathrm{b}}$ \\
\hline Hubal & $23.86^{\text {bcde }}$ & $5.54^{\mathrm{abc}}$ & $6.28^{\mathrm{c}}$ & $44.70^{\mathrm{ab}}$ \\
\hline Klif & $25.20^{\mathrm{g}}$ & $5.23^{\mathrm{a}}$ & $6.28^{\mathrm{c}}$ & $44.95^{\mathrm{ab}}$ \\
\hline Marych & $24.98^{\text {ef }}$ & $5.48^{\mathrm{abc}}$ & $6.30^{\mathrm{c}}$ & $38.61^{\mathrm{a}}$ \\
\hline Milwa & $23.58^{\mathrm{bc}}$ & $6.16^{\mathrm{d}}$ & $5.70^{\mathrm{ab}}$ & $47.35^{\mathrm{b}}$ \\
\hline Model & $23.49^{\mathrm{abcd}}$ & $5.36^{\mathrm{ab}}$ & $6.25^{\mathrm{bc}}$ & $46.78^{\mathrm{b}}$ \\
\hline Muza & $23.62^{\mathrm{bcd}}$ & $5.37^{\mathrm{b}}$ & $5.59^{\mathrm{a}}$ & $48.31^{\mathrm{b}}$ \\
\hline Pomorska & $23.56^{\mathrm{abcd}}$ & $6.13^{\mathrm{d}}$ & $6.35^{\mathrm{c}}$ & $42.39^{\mathrm{ab}}$ \\
\hline Roch & $24.28^{\text {bcdef }}$ & $5.79^{\mathrm{bcd}}$ & $6.05^{\mathrm{abc}}$ & $44.07^{\mathrm{ab}}$ \\
\hline Sokolik & $23.39^{\mathrm{ab}}$ & $5.88^{\mathrm{cd}}$ & $6.17^{\mathrm{bc}}$ & $46.09^{\mathrm{b}}$ \\
\hline Turnia & $22.35^{\mathrm{a}}$ & $5.59^{\mathrm{abc}}$ & $6.35^{\mathrm{c}}$ & $47.01^{\mathrm{b}}$ \\
\hline Wiato & $24.77^{\text {def }}$ & $5.56^{\mathrm{abc}}$ & $6.11^{\mathrm{abc}}$ & $44.56^{\mathrm{ab}}$ \\
\hline Mean & $\mathbf{2 3 . 9 3}$ & $\mathbf{5 . 6 1}$ & $\mathbf{6 . 6 1}$ & $\mathbf{4 5 . 2 2}$ \\
\hline
\end{tabular}

*see the explanation under Table 1

of the protein content was relatively small, whereas its higher concentration characterized was recorded for cultivars Kavalir and Ezop (Table 7). However, fodder cultivars Wiato, Klif, and Marych showed the highest protein content, while cvs. Turnia and Sokolik the lowest (Table 10). The mean protein content in the seeds of

Table 11. Nutrient contents in fodder cultivars of field pea depending on the cultivation region (\% DM).

\begin{tabular}{|c|c|c|c|c|}
\hline Cultivar & $\begin{array}{c}\text { Crude } \\
\text { protein }\end{array}$ & $\begin{array}{c}\text { Crude } \\
\text { fibre }\end{array}$ & Sugars & Starch \\
\hline Białogard & $23.42^{* c}$ & $4.80^{\mathrm{a}}$ & $6.07^{\mathrm{ab}}$ & $46.57^{\mathrm{b}}$ \\
\hline Bobrowniki & $23.66^{\mathrm{bc}}$ & $6.17^{\mathrm{c}}$ & $6.14^{\mathrm{ab}}$ & - \\
\hline Cicibór Duży & $25.04^{\mathrm{d}}$ & $5.01^{\mathrm{ab}}$ & $6.36^{\mathrm{ab}}$ & $43.07^{\mathrm{a}}$ \\
\hline Głodowo & $23.51^{\mathrm{d}}$ & $6.43^{\mathrm{c}}$ & $6.13^{\mathrm{a}}$ & - \\
\hline Kościelec & $25.00^{\mathrm{d}}$ & $6.45^{\mathrm{c}}$ & $6.14^{\mathrm{ab}}$ & - \\
\hline Marianowo & $22.60^{\mathrm{ab}}$ & $4.89^{\mathrm{ab}}$ & $6.12^{\mathrm{ab}}$ & - \\
\hline Pawłowice & $25.35^{\mathrm{d}}$ & $6.30^{\mathrm{c}}$ & $6.36^{\mathrm{ab}}$ & - \\
\hline Ruska Wieś & $23.27^{\mathrm{abc}}$ & $4.86^{\mathrm{b}}$ & $6.01^{\mathrm{ab}}$ & - \\
\hline Świebodzin & $23.65^{\mathrm{bc}}$ & $5.00^{\mathrm{ab}}$ & $6.33^{\mathrm{b}}$ & - \\
\hline Tomaszów Bol. & $25.40^{\mathrm{d}}$ & $4.83^{\mathrm{a}}$ & $6.33^{\mathrm{b}}$ & $46.02^{\mathrm{b}}$ \\
\hline Wyczechy & $22.91^{\mathrm{abc}}$ & $4.79^{\mathrm{a}}$ & $5.97^{\mathrm{ab}}$ & - \\
\hline Mean & $\mathbf{2 3 . 9 8}$ & $\mathbf{5 . 4 1}^{\mathrm{a}}$ & $\mathbf{6 . 1 8}$ & $\mathbf{4 5 . 2 2}^{\mathrm{a}}$ \\
\hline
\end{tabular}

*see the explanation under Table 1 
Table 12. Correlation coefficients between nutrient contents in fodder pea seeds and some agrotechnical factors.

\begin{tabular}{|c|c|c|c|c|c|}
\hline & $\begin{array}{c}\text { Crude } \\
\text { protein }\end{array}$ & $\begin{array}{c}\text { Crude } \\
\text { fibre }\end{array}$ & Tannins & Sugars & Starch \\
\hline Crude fibre & -0.037 & & & & \\
\hline Tannins & $\mathbf{- 0 . 1 2 4}$ & -0.007 & & & \\
\hline Sugars & $\mathbf{0 . 2 1 3}$ & $\mathbf{- 0 . 1 7 0}$ & $\mathbf{0 . 1 3 6}$ & & \\
\hline Starch & $\mathbf{- 0 . 3 9 2}$ & 0.174 & -0.075 & -0.190 & \\
\hline Soil complex & $\mathbf{0 . 1 3 3}$ & $\mathbf{- 0 . 5 2 9}$ & -0.139 & 0.089 & 0.181 \\
\hline Soil pH & $\mathbf{- 0 . 1 4 6}$ & $\mathbf{- 0 . 1 4 8}$ & $\mathbf{0 . 1 9 7}$ & $\mathbf{0 . 1 6 3}$ & -0.460 \\
\hline Forecrop & $\mathbf{0 . 1 8 9}$ & $\mathbf{- 0 . 2 1 3}$ & -0.004 & $\mathbf{0 . 2 6 0}$ & -0.107 \\
\hline Precipitation & $\mathbf{- 0 . 1 1 6}$ & $\mathbf{- 0 . 1 8 9}$ & 0.103 & -0.015 & -0.008 \\
\hline
\end{tabular}

*- bold letters means significant differences

fodder cultivars was higher than in edible cultivars. Significantly more of this component was recorded in the seeds of all cultivars from southern Poland (Tables $8,11)$. Canbolat et al. [31] noted significant differences in the chemical composition of the studied pea genotypes depending on the color of seeds and flowers. According to these authors, the protein content in the dark seeds was higher than in light ones. According to Fordoński et al. [12], protein content in the pea seeds equaled $20.7 \%$ of dry matter. Canbolat et al. [31] indicated that the energy value of pea seeds is similar to the energy value of soybean. Rybiński et al. [30], evaluating the protein content of pea seeds, recorded its highest amount in cv. Moreover, this author found that pea seeds have a low fat content, whereas it was higher content found in $\mathrm{cv}$. Medal and strain PRH 179. The authors emphasized the high content of unsaturated fatty acids, i.e., of linolenic acid belonging to the group of EFA (essential fatty acids). In our own study, the lowest content of crude fibre in all cultivars was recorded in northern, more in central, and the largest in southeastern Poland (Tables 8, 11). Fordoński et al. [32] reported that the seeds of fodder and edible peas cultivated in northern Poland characterized similar content of crude protein, crude fibre, crude fat, and ash. The tested cultivars had varied fibre content. It was generally higher in fodder cultivars. Among edible cultivars, its lowest amounts were recorded for Mecenas, while among fodder ones for Klif, Gwarek, Model, and Muza. The highest amounts of crude fibre were found for edible cvs. Cysterski, Santana, and Bohun, and for fodder cvs. Pomorska and Milwa (Tables 7, 10). According to Kotecki [33], cv. Ergo contained more fibre and protein, while cv. Diament less. This author also found that the change of crop density did not have a major impact on the content of essential nutrients. The research conducted by Wang et al. [34] demonstrated significant differences in the content of protein, and crude fibre in pea seeds depending on the system and location of cultivation.

Most of the evaluated cultivars had a similar soluble sugar content; it was only slightly smaller in cvs. Wenus (edible) and Muza (fodder), and higher in cvs. Zekon (edible), Klif, Hubal, Marych, Pomorska, and Turnia (fodder) (Tables 7, 10). The most favorable conditions for sugar accumulation occurred in southern Poland, while the least favorable were in northeastern and northern Poland (Tables 8, 11). The seeds of edible cultivars Bohun, Mentor, Lasso, Ezop, and Wenus accumulated the most starch, and the seeds of cv. Terno the least. The location of pea cultivation had little effect on starch content, although its bigger amounts were generally recorded in eastern Poland (Table 8). However, fodder pea cultivars showed a relatively small variability in starch content, with the lowest amount being recorded in cv. Marych (Table 8). According to Mikić et al. [35], tannins are most often present in the seeds of cultivars of colorful flowers that may be used in the production of feed, although they are often characterized by a lower digestibility of proteins compared to other cultivars of pea. However, according to those authors [35], the seeds of cultivars without tannins have a wide application both in human and animal feeding.

Correlation analysis showed that the protein content in the seeds of all tested edible pea cultivars was positively affected by soil $\mathrm{pH}$ and forecrop, after which pea was grown (significant differences; Table 9). Kotecki [33] found that the amount of this component in pea seeds depended mainly on weather conditions, while to a lesser extent on the genetic characteristics of a cultivar or plant density per unit area. Soil complex has a positive effect on crude protein content in the seeds. Improvement of soil $\mathrm{pH}$ has a positive effect on sugars and negative on crude fibre content. Forecrop, after which field pea was grown was positively correlated with crude protein and sugar content. Increasing the sum of precipitation caused a decrease of crude fibre, sugars, and starch content in the pea seeds. The crude protein content in the seeds of fodder peas was negatively affected by the content of tannin and starch, while positively correlated with the amount of soluble sugars (Table 12). The crude fibre content in the seeds of all the tested cultivars was significantly reduced by the sugar content. The amount of tannins in the seeds accumulated in fodder pea seed cultivars increases together with higher sugar content. All evaluated agronomic factors such as soil complex, soil $\mathrm{pH}$, forecrop, and sum of precipitations negatively affected crude fibre content in fodder cultivars of field pea seeds (significant correlations). Soil $\mathrm{pH}$ negatively affected content of crude protein while positively affecting content of tannins and sugars. Forecrop has a positive effect on crude protein and sugar content. Increasing the sum of total precipitation in vegetation season caused a decrease of crude fibre and crude protein contents.

\section{Conclucions}

1) Regardless of the agro-ecological conditions, a lower amount of protein was recorded for faba bean cultivars: Sonet, Opitmal, and Granit, while higher 
for Kasztelan, Leo, Olga, Bobas, Amulet, and Albus. The lowest crude fibre content was found in cultivars Bobas and Granit. In southern Poland the mean crude fibre and crude protein contents were higher than in the northern and northwestern parts of the country.

2) The average protein content in field pea seeds was higher in the fodder cultivars than in the edible ones. A larger concentration of crude protein in edible cultivars was recorded in cvs. Kavalir and Ezop. Fodder cultivars Wiato, Klif, and Marych had the highest protein content, and Turnia and Sokolik the smallest. The region of pea cultivation influenced protein and fibre contents: in southern Poland, pea cultivars contained the most crude protein and crude fibre compared with other regions.

3) The seeds of edible cultivars Wenus, Bohun, Mentor, Lasso, and Ezop accumulated the most starch, while the seeds cv. Terno accumulated the least. The region of pea cultivation had little impact on its quantity. Fodder cultivars showed relatively little variability in starch content.

4) Fodder pea cultivars exhibited a higher fibre content compared with edible ones. The least fibre was recorded for edible cv. Klif, and fodder cvs. Klif, Gwarek, Model, Muza, while the most, respectively, were found in edible Cysterski, Santana, Bohun, and fodder cvs. Pomorska and Milwa. The smallest content of fibre among all the cultivars was noted in the northern, while the largest in the southeastern regions of Poland. Most cultivars of field pea showed a similar sugar content. The most favorable conditions of sugar accumulation occurred in the southern part and the least in the northeastern and northern parts of the country.

\section{References}

1. VILARIÑO M., MÉTAYER J.P., CRÉPON K., DUC G. Effects of varying vicine, convicine and tannin contents of faba bean seeds (Vicia faba L.) on nutritional values for broiler chicken. Animal Feed Science and Technology, 150, 1-2, 114, 2009.

2. NALLE C.L., RAVINDRAN V., RAVINDRAN G. Evaluation of faba beans, white lupines and peas as protein source in broiler diets. International Journal of Poultry Science, 9 (6), 567, 2010.

3. JEZIERNY D., MOSENTHIN R., BAUER E. The use of grain legumes as a protein source in pig nutrition: A review. Animal Feed Science and Technology, 157, 111, 2010.

4. OSEK M., MILCZAREK A., KLOCEK B., TURYK Z., JAKUBOWSKA K. Effectiveness of mixtures with the Fabaceae seeds in broiler chicken feeding, Annales UMCS, sec. EE, Zootechnica, 67, 77, 2013.

5. TUFARELLI V., LAUDADIO V. Feeding of dehulled-micronized faba bean (Vicia faba var. minor) as substitute for soybean meal in guinea fowl broilers: Effect on productive performance and meat quality. Asian-Australasian Journal of Animal Science, 28 (10), 1471, 2015.

6. KSIĘŻAK J., STANIAK M., BOJARSZCZUK J. The regional differentiation of legumes cropping area in Po- land within 2001-2007. Polish Journal of Agronomy, 1, 25, 2009.

7. CAMPOS-VEGA R., LOARCA-PINA G., OOMAH B.D. Minor components of pulses and their potential impact on human health. Food Research International, 43(2), 461, 2010. https://doi.org/10.1016/j.foodres.2009.09.004

8. CHAPAGAIN T., RISEMAN A. Nitrogen and carbon transformations, water use efficiency and ecosystem productivity in monocultures and wheat-bean intercropping systems. Nutrient Cycling in Agroecosystems 101 (1), 107, 2015. https://doi.org/10.1007/s10705-014-9647-4

9. MATEOS-APARICIO I., REDONDO-CUENCA A., VILLANUEVA-SUAREZ M., ZAPATA-REVILLA M., TENORIO-SANZ M. Pea pod, broad bean pod and okara, potential sources of functional compounds. LWT - Food Science and Technology, 43 (9), 1467, 2010.

10. VIOQUE J., ALAIZ M., GIRON-CALLE J. Nutritional and functional properties of Vicia faba protein isolates and related fractions. Food Chemistry, 132 (1), 67, 2012. https://doi.org/10.1016/j.foodchem.2011.10.033.

11. DURANTI M. Grain legume proteins and nutraceutical properties. Fitoterapia, 77 (2), 67, 2006. https://doi. org/10.1016/j.fitote.2005.11.008.

12. FORDOŃSKI G., PSZCZÓŁKOWSKA A., KRZEBIETKE S., OLSZEWSKI J., OKORSKI A. Yield and mineral composition of seeds of leguminous plants and grain of spring wheat as well as their residual effect on the yield and chemical composition of winter oilseed rape seed. Journal of Elementology, 20 (4), 827, 2015.

13. FLOREK J., CZERWIŃSKA-KAYZER D., JERZAK A. Current state of production and use of leguminous crops. Fragmenta Agronomica, 29 (4), 45, 2012 [In Polish].

14. SARAH A., ABUSIN E., HASSAN A.B., BABIKER E.E.. Nutritional evaluation of cooked faba bean (Vicia faba L.) and white bean (Phaseolus vulgaris L.) cultivars. Australian Journal of Applied Science, 3 (3), 2484, 2009.

15. MEKKEI M.E. Effect of intra-row spacing and seed size on yield and seed quality of faba bean (Vicia faba L.) International Journal of Agriculture and Crop Sciences. Available online at www.ijagcs.com IJACS/2014/7-10/665-670 ISSN 2227-670X (C2014.

16. HENDAWEY M.H., YOUNES A.M.A. Biochemical evaluation of some faba bean cultivars under rainfed conditions at El-heikh Zuwayid. Annals of Agricultural Sciences, 5892, 183, 2013. https://doi.org/10.1016/j.aoas.2013.07.010

17. WOŹNIAK A., SOROKA M., STĘPNIOWSKA A., MAKARSKI B. Chemical composition of pea (Pisum sativum L.) seeds depending on tillage systems. Journal of Elementology, 19 (4), 1143, 2014.

18. MILCZAREK A., OSEK M. Partial replacement of soya bean with low-tannin faba bean varieties (Albus or Amulet): effects on growth traits, slaughtering parameters and meat quality of Pulawska pigs. Annals of Animal Science, 16 (2), 477, 2016.

19. KSIĘŻAK J. The dynamics of nutrient uptake by traditional and self-finishing varieties of horse bean between flavoring and full maturity. Monografie i Rozprawy Naukowe, IUNG -PIB Puławy, 5, 95, 2002.

20. PISULEWSKA E., HANCZAKOWSKI P., SZYMCZYK B., ERNEST T., KULIG B.,Comparison of chemical composition, content of anti-nutritive substances and nutritive value of seeds of nine faba bean varieties (Vicia faba L.) cultivated in two growing seasons. Roczniki Naukowe Zootechniki, 23 (2), 253, 1996 [In Polish].

21. MUSALAM I.W., AL-KARAKI G., EREIF K.I., TAWAHA, A.M. Chemical composition of faba bean cul- 
tivars under rained and irrigation conditions. International Journal of Agriculture and Biology, 6 (2), 359, 2004.

22. DESCRIPTIVE LIST OF AGRICULTURAL PLANT VARIETIES. Research Centre for Cultivar Testing Słupia Wielka, 2010.

23. SWINE NUTRITION STANDARDS. Nutritional recommendations and nutritional value of feed for pigs Grela E.R. i Skomiał (red.), wyd. II, Instytut Fizjologii i Żywienia Zwierząt im. J. Kielanowskiego, PAN Jabłonna, 2015.

24. SZPUNAR KROK E., BOBRECKA-JAMRO D., TOBIASZ-SALACH R., KUBIT P. Chemical composition of naked grains oat and faba bean seeds in pure sowing and mixtures. Fragmenta Agronomica, 26, 152, 2009 [In Polish].

25. HANCZAKOWSKA E., ŚWIĄTKIEWICZ M. Legume seeds and rapeseed press cake as replacers of soybean meal in feed for fattening pigs. Annales of Animal Science, 14, 921, 2014.

26. KIARIE E., LOPEZ P., FUREDIC., NYACHOTI C.M. Amino acids and energy utilization in zero-tannin faba bean and co-fermented wheat and corn dried distillers grains with solubles fed to growing pigs. Journal of Animal Science, 91, 1728, 2013.

27. ABUSIN S.A.E., HASSAN A.B., BABIKER E.E. Nutritional evaluation of cooked faba bean (Vicia Faba L.) and white bean (Phaseolus Vulgaris L.) cultivars. Australian Journal of Basic and Applied Sciences, 3 (3), 2484, 2009.

28. EL-SABER, M.M.M.,. Biochemical Studies on Faba Bean Under Rainfed at Maryout Condition. MSc Thesis, Biochemistry Department, Faculty of Agriculture, Zagazig University, Egyp, 2010.
29. JASIŃSKA Z., KOTECKI A. Characteristics of field bean strains and cultivars with terminal inflorescence. Part. II. Chemical composition and nutritional value of grain. Zeszyty Naukowe AR Wrocław, Rolnictwo, 347, 173, 1998 [In Polish].

30. RYBIŃSKI W., STARZYCKI M., RUSINEK R., BOCIANOWSKI J., SZOT B. Variation of legume seed's chemical composition and resistance to mechanical damage. Biuletyn IHAR, 268, 193, 2013 [In Polish].

31. CANBOLAT O., TAMERAND E., ACIKGOZ E. Chemical Composition, Metabolizable Energy and Digestibility in Pea Seeds of Differing Testa and Flower Colors, Journal of Biological and Environmental Science, 1 (2), 59, 2007.

32. FORDOŃSKI G., GRONOWICZ Z., PAPROCKI S. The effect of seeding rate on yield and nutritive value of new pea cultivars. Acta Academiae Agricutulturae Technicae Olstenensis, 45, 157, 1988 [In Polish].

33. KOTECKI A. The influence of sowing density on the development and yielding of several pea cultivars.. Zeszyty Naukowe Akademii Rolniczej we Wrocławiu, 253, 57, 1994 [In Polish].

34. WANG N., HATCHER D.W., WARKENTIN T.D., TOEWS R. Effect of cultivar and environment on physiochemical and cooking characteristics of field pea (Pisum sativum L.). Food Chemistry, 118, 109, 2010.

35. MIKIĆ A., PERIĆ V., ĐORĐEVIĆ V., SREBRIĆ M., MIHAILOVIĆ V. Anti-nutritional factors in some grain legumes. Biotechnology in Animal Husbandry, 25 (5-6), 1181, 2009. 